\title{
Effect of Colchicine on Total Antioxidant Capacity, Antioxidant Enzymes and Oxidative Stress Markers in Patients with Knee Osteoarthritis
}

\author{
Metin Erden ${ }^{1 *}$, Levent Ediz ${ }^{2}$, Özcan Hız ${ }^{2}$, Yasin Tuluce ${ }^{3}$, Halil Ozkol ${ }^{3}$, Murat Toprak ${ }^{1}$ \\ Fahrettin Demirdag ${ }^{4}$ \\ ${ }^{1}$ Physical Medicine and Rehabilitation Department, District Education and Research Hospital, Van, Turkey; ${ }^{2}$ Physical Medicine and \\ Rehabilitation Department, Yuzuncu Yil University Medical Faculty, Van, Turkey; ${ }^{3}$ Medical Biology Department, Medical Faculty, \\ Yuzuncu Yil University, Van, Turkey; ${ }^{4}$ Physical Medicine and Rehabilitation Department, District Education and Research Hospital, \\ Elazig, Turke. \\ Email: ${ }^{*}$ r.metinerden@gmail.com
}

Received May $28^{\text {th }}, 2012$; revised June $30^{\text {th }}, 2012$; accepted July $15^{\text {th }}, 2012$

\begin{abstract}
Background/Aim: We aimed to investigate the effects of colchicine on clinical recovery, as well as oxidative stress markers and total antioxidant capacity (TAC) in whole blood of patients with knee osteoarthritis (OA). Materials and Methods: Sixty patients with grade $2-3$ knee OA according to ACR knee OA criteria whom examination of the knee joint synovial fluid by polarized light microscopy demonstrated CPDD crystals existence were included in the study. Fifty healthy subjects were included as a control group. Patients were divided randomly into two groups. The first group (paracetamol group) was given only paracetamol 3 gr daily p.o and the second group (colchicine and paracetamol group) was given colchicine $1.5 \mathrm{gr}$ and paracetamol $3 \mathrm{gr}$ daily p.o for six months. For outcome measures WOMAC and VAS were used. Superoxide dismutase (SOD), Catalase (CAT) ezyme activities and Glutathione (GSH) and Malondialdehyde (MDA) levels and TAC all were measured. Results: WOMAC scores were improved in both patient groups compared with pre-treatment evaluation $(\mathrm{p}<0.025)$. WOMAC morning stiffness scores were significantly more improved in colchicine group compared with paracetamol group $(p>0.05)$. TAC was signifcantly increased only in colchicine/paracetamol group. Oxidant parameter MDA levels were significantly decreased in both paracetamol group and colchicine/paracetamol group. CAT, SOD enzyme activities and GSH levels did not change before and after treatment protocols in both patient groups. Conclusion: Both paracetamol $3000 \mathrm{mg} / \mathrm{day}$ and $3000 \mathrm{mg}$ paracetamol plus $1.5 \mathrm{gr} /$ day colchicine is effective in the treatment of patients with knee osteoarthritis. But the addition of colchicine to paracetamol produced significantly greater symptomatic benefit than paracetamol alone. Our study also showed that colchicine lowers whole blood MDA which is a lipid peroxidation compound and elevates TAC levels in patients with knee OA. This may show probable disease modifying effect of colchicine in knee OA which require further long period laboratory and radiologic investigations.
\end{abstract}

Keywords: Knee Osteoarthritis; Colchicine; Paracetamol; Total Antioxidant Capacity; Malondialdehyde; Oxidative Stres

\section{Introduction}

Osteoarthritis (OA) is a chronic degenerative disorder of multifactorial etiology characterized by loss of articular cartilage and changes in the underlying bone at the joint margins. OA is a leading cause of chronic joint pain and disability at older ages, largely due to knee and hip involvement. The etiology of OA is unclear. Mechanical, biochemical, metabolic, endocrine, genetic and environmental factors seem to play a role [1].

The incidence of osteoarthritis increases steeply after

"Corresponding author.
50 years of age and the proportion of elderly people in the population continues to increase. More than $13 \%$ of Americans aged 55 to 64 years, and more than $17 \%$ of Americans aged 65 to 74 years, have pain and functional limitations related to knee OA [2].

Basic calcium phosphate (BCP) and calcium pyrophosphate dihydrate (CPPD) crystals are commonly found in osteoarthritic joints. These crystals have been found in the synovial fluid of $60 \%$ of patients with knee OA and over $90 \%$ of a small group of patients with grade- 4 OA $[3,4]$.

Clinical and experimental data suggest a pathogenic 
role for calcium-containing crystals in cartilage destruction and OA development and progression [5].

Oxidative demage to collagen (oxidative DNA demage etc.) has also important role in etiopathogenesis of OA. Decreased joint fluid antioxidants and increased reactive oxygen species (ROS) have been shown in osteoarthritic joints compared to joints with macroscropically intact cartilage and subacute injury. ROS induced by IL- $1 \beta$ and TNF- $\alpha$ can directly cleave proteoglycans and collagen, modulate signaling pathways and activate MMPs, as well as alter chondrocyte apoptosis and cellular synthetic activity [4,6-24].

Recently there has been increase in trials on disease/ structure-modifying agents in OA (e.g. MMP inhibitors, glucosamine or chondritin sulphate, colchicine, strontium ranelate, biphosphonates and diacetylrhein/diacerein). This is an area of active research at present. Preclinical studies of potential disease modifying agents are promising. In clinical studies, generally, radiological joint space narrowing or width have been used as the structural outcome measure in these trials. More advanced compositional MRI techniques, and laboratory cartilage markers might enable to detect early cartilage changes before radiographic joint space narrowing becomes evident [25-27].

Furthermore there are studies in the literature, on slow acting disease/structure-modifying effects of colchicine in knee OA. The rationale using colchicine as disease/ structure-modifying drug to manage OA resides in the fact that calcium-containing crystals are frequently seen in OA $(3,4)$, and colchicine has been shown to be beneficial in preventing calcium crystal-induced inflammation (pseudogout). When used to treat gouty arthritis, colchicine is believed to work by inhibiting microtubule dependent cell infiltration and IL- $1 \beta$ production. However, in vitro and in vivo, colchicine also reduces monosodium urate (MSU)-induced ROS production by neutrophils [28-31].

Taking into account that increased incidence of calcium-containing crystals (from $60 \%$ upto $90 \%$ ), decreased joint fluid antioxidants and increased reactive oxygen species (ROS) in osteoarthritic joints, we aimed to investigate the effects of colchicine on clinical recovery, oxidative stress markers and total antioxidant capacity (TAC) in whole blood of patients with knee osteoarthritis.

\section{Materials and Methods}

Sixty patients with grade 2 - 3 knee osteoarthritis according to ACR knee OA criteria whom examination of the knee joint synovial fluid by polarized light microscopy demonstrated CPDD crystals existence were included in the study. Fifty healthy subjects who have similar demographic characteristics with patient group were included as a control group. The study protocol, consent form and all recruitment materials were approved by the ethical Board of the University of Yuzuncu Yil University, Van, Turkey, and the study was conducted in accordance with the Declaration of Helsinki. Patients were divided randomly into two groups. The first group (paracetamol group) was given only paracetamol 3 gr daily p.o and the second group (colchicine and paracetamol group) was given colchicine $1.5 \mathrm{gr}$ and paracetamol 3 gr daily p.o.

The patients with diabetes, gouty arthritis, secondary knee osteoarthritis due to inflammatory joint diseases, a history of intraarticular corticosteroid and/or hyaluronic acid injections, and a history of oral glucosamine, colchicine use during the last 6 months, chronic alchohol consumption, chronic liver and kidney diseases, smokers, a history of antioxidant vitamin and/or mineral use during the last six months were excluded.

One of the primary clinical outcome measures was change in the WOMAC-Western Ontario and McMaster Universities Arthritis Index. The WOMAC has been used extensively in the quantitative assessment of knee OA, and has been proven to be effective in assessing functionality, pain in patients suffering from knee OA. Turkish WOMAC has been found valid and reliable [32].

Another clinical outcome measure was 100 mm VAS (visual analog scale) for pain measuremet.

Before drug treatment start and after the last treatment dose (6 months later), $7 \mathrm{~mL}$ sample of venous blood was taken in the morning before breakfast from each patient and control subjects. Serum samples were stored at $-20^{\circ} \mathrm{C}$ until analysis.

\subsection{Oxidant/Antioxidant Status Analysis}

2.1.1. Superoxide Dismutase (SOD) Enzyme Analysis SOD enzyme activity was measured using Genesys 10 UV-VIS Scanning spectrophotometer by Randox-Ransod enzyme kit at $505 \mathrm{~nm}$ and $37^{\circ} \mathrm{C}$.

\subsubsection{Catalase (CAT) Enzyme Activity Determination} Catalase enzyme activity was measured by reading the absorbance with a spectrophotometer (Genesys $10 \mathrm{UV}$ Scanning UV/VIS Spectrophotometer) set at $240 \mathrm{~nm}$ based on Aebi method.

\subsubsection{Glutathion (GSH) Determination}

GSH samples were measured by using a spectrofluorimetry (Jasco 6000 USA) with excitation at $345 \mathrm{~nm}$ and emission at $425 \mathrm{~nm}$.

\subsubsection{Total Antioxidant Capacity (TAC)}

Ready-kit was used according to the measurement method.

\subsubsection{Malondialdehyde (MDA) Determination}

Levels of MDA which is a peroxidation product of lipid 
metabolism were analyzed spectrofluorimetrically, using the modified method by Hegde et al. [33]. The MDA was measured by a spectrofluorimetry (Jasco 6000, USA), with excitation at $520 \mathrm{~nm}$ and emission at $555 \mathrm{~nm}$.

All outcome measures were performed before colchicine tretment and just after of colchicine treatmet finish (6 months later).

\subsection{Statistical Analysis}

Results were expressed as mean and standard deviation (SD). Statistical analysis was carried out using the SPSS program (version 11.5 software, SPSS Inc. Chicago, Illinois, USA). For the comparison of groups, independent student $t$ test, ANOVA, Kruskal Wallis and MannWhitney U test were used. Categorical variables were evaluated with Pearson's chi-square test. Within the same group comparisons in terms of the difference between pretreatment and posttreatment measurements were investigated by the dependent t-test or Wilcoxon signed rank test. $P$ values of less than 0.05 were regarded as significant.

\section{Results}

Demographic data of the groups were presented in Table
1. There was no significant difference between groups in terms of demographic variables.

VAS scores according to patient and doctor evaluations of the tretment groups were presented in Table 2. Only Colchicine group showed statistically significant change after the treatment in terms of VAS scores compared with pre-treatment evaluation.

Pre and post-treatment WOMAC scores of the patient groups were presented in Table 3. Post-treatment WOMAC scores were improved in both patient groups compared with pre-treatment evaluation $(p<0.025)$. WOMAC morning stiffness scores were significantly more

Table 1. Demographic properties of the groups.

\begin{tabular}{lcccc}
\hline Variables & Control & Paracetamol & $\begin{array}{c}\text { Colchicine/ } \\
\text { Paracetamol }\end{array}$ & p-Value \\
\hline Age & $56.7 \pm 6.7$ & $55.4 \pm 6.2$ & $57.6 \pm 7.1$ & 0.452 \\
Gender & & & & 0.895 \\
Male (n) & $19(38.0 \%)$ & $13(43.3 \%)$ & $12(40.0 \%)$ & \\
Female (n) & $31(62.0 \%)$ & $17(56.7 \%)$ & $18(60.0 \%)$ & \\
Kellgren Grade & & & & 0.598 \\
Grade II & - & $11(36.7 \%)$ & $13(43.3 \%)$ & \\
Grade III & - & $19(63.3 \%)$ & $17(56.7 \%)$ & \\
\hline
\end{tabular}

Table 2. Pre-treatment and post-treatment VAS scores of the patient groups.

\begin{tabular}{|c|c|c|c|c|c|}
\hline Variables & Pre-treatment (mean(SD)) & Post-treatment (mean(SD)) & p-Value ${ }^{\mathrm{a}}$ & Change (mean(SD)) & p-Value ${ }^{b}$ \\
\hline $\mathrm{VAS}_{\text {patient }}$ & & & & & 0.645 \\
\hline Paracetamol & $77.5(17.25)$ & $74.0(17.25)$ & 0.060 & $-4.0(8.00)$ & \\
\hline Colchicine/Par & $79.0(11.50)$ & $76.0(14.00)$ & 0.010 & $-4.0(8.50)$ & \\
\hline VAS $_{\text {doctor }}$ & & & & & 0.662 \\
\hline Paracetamol & $75.5(7.25)$ & $74.0(15.25)$ & 0.043 & $-3.0(9.25)$ & \\
\hline Colchicine/Par & $73.0(17.25)$ & $68.0(15.50)$ & 0.033 & $-3.5(12.00)$ & \\
\hline
\end{tabular}

${ }^{\mathrm{a}}$ Comparisons between pre-treatment and post-treatment within the same groups, according to the Bonferroni correction $\mathrm{p}$ value $<0.025$ was considered statistically significant for the results; ${ }^{\mathrm{b}}$ Comparisons between the groups in terms of treatment-related change, p-value $<0.05$ was considered statistically significant for the results.

Table 3. Pre-teratment and post-treatment WOMAC scores of the patient groups.

\begin{tabular}{|c|c|c|c|c|c|}
\hline Variables & Pre-treatment (mean(SD)) & Post-treatment (mean(SD)) & $\mathrm{p}-$ Value $^{\mathrm{a}}$ & Change (mean(SD)) & p-Value ${ }^{b}$ \\
\hline Pain & & & & & 0.988 \\
\hline Paracetamol & $11.5(4.00)$ & $11.0(3.25)$ & 0.008 & $-1.0(2.25)$ & \\
\hline Colchicine/Par & $11.0(5.00)$ & $9.5(4.25)$ & 0.011 & $-1.0(3.00)$ & \\
\hline Morning stiffness & & & & & 0.039 \\
\hline Paracetamol & $1.0(2.25)$ & $1.0(2.00)$ & 0.206 & $0.0(0.00)$ & \\
\hline Colchicine/Par & $3.0(4.25)$ & $2.0(3.25)$ & 0.005 & $-1.0(1.25)$ & \\
\hline Physical activity & & & & & 0.755 \\
\hline Paracetamol & $38.0(11.00)$ & $36.5(14.25)$ & 0.027 & $-2.5(6.50)$ & \\
\hline Colchicine/Par & $34.5(17.00)$ & $31.0(13.25)$ & 0.021 & $-2.0(8.25)$ & \\
\hline Total & & & & & 0.280 \\
\hline Paracetamol & $12.2(6.50)$ & $11.5(6.08)$ & 0.002 & $-1.0(1.91)$ & \\
\hline Colchicine/Par & $13.3(8.75)$ & $11.9(5.56)$ & 0.002 & $-1.0(2.85)$ & \\
\hline
\end{tabular}

${ }^{a}$ Comparisons between pre-treatment and post-treatment within the same groups, according to the Bonferroni correction $\mathrm{p}$-value $<0.025$ was considered statistically significant for the results; ${ }^{b}$ Comparisons between the groups in terms of treatment-related change, p-value $<0.05$ was considered statistically significant for the results. 
improved in colchicine group compared with paracetamol group $(\mathrm{p}>0.05)$.

Baseline oxidant and anti-oxidant levels of the control group and the treatment groups were presented in Table 4. There were statistically significant differences between control group and treatment groups in terms of baseline MDA levels $(\mathrm{p}<0.001)$. Baseline MDA levels were higher in treatment groups than control group.

Comparisons of pre-treatment and post-treatment oxidant and antioxidant levels of the patient groups were presented in Table 5. Total antioxidant capacity (TAC) was signifcantly increased only in colchicine/paracetamol group. Oxidant parameter MDA levels were significantly decreased in both paracetamol group and colchicine/paracetamol group. Catalase (CAT), superoxide dismutase (SOD) enzyme activities and glutathione (GSH) levels did not change before and after treatment protocols in both patient groups.

\section{Discussion}

In this study, the effects of colchicine on whole blood oxidant load, antioxidant capacity and clinical recovery in patients with $\mathrm{OA}$ were investigated. In the current study we found that baseline MDA levels were higher in patients with OA than healthy control group. Baseline SOD, CAT enzyme activities, GSH, and TAC levels were not differed between groups. When compared to baseline MDA levels, post-treatment MDA levels significantly decreased in only the group receiving colchicine/ paracetamol. Post-treatment TAC levels were also increased significantly in only the group receiving colchicine/paracetamol. No significant changes were observed in post-treatment SOD, CAT enzyme activities and GSH levels in both groups.

As well as colchicine treated group showed more improvement in clinical recovery parameters such as WOMAC and VAS scores than control and paracetamol treated groups. Our clinical recovery results are in accordance with previous studies by Ediz L. et al. and Das K. et al. [28,34].

Basic calcium phosphate (BCP) and calcium pyrophosphate dihydrate (CPPD) crystals are commonly found in osteoarthritic joints. These crystals have been found in the synovial fluid of $60 \%$ of patients with knee OA and over $90 \%$ of a small group of patients with grade- 4 OA

Table 4. Pre-treatment (baseline) laboratory findings of control and treatment Groups.

\begin{tabular}{ccccc}
\hline Variables & Control & Paracetamol & $\begin{array}{c}\text { Colchicine/ } \\
\text { Paracetamol }\end{array}$ & p-Value \\
\hline CAT & $56.4(18.30)$ & $54.8(13.02)$ & $59.2(18.97)$ & 0.328 \\
GSH & $0.98(0.09)$ & $1.0(0.17)$ & $0.99(0.06)$ & 0.743 \\
TAC & $25.0 \pm 5.78$ & $24.0 \pm 7.25$ & $21.6 \pm 5.31$ & 0.081 \\
MDA & $5.7(1.11)^{\mathrm{a}, \mathrm{b}}$ & $6.6(1.32)^{\mathrm{a}}$ & $6.1(1.94)^{\mathrm{b}}$ & $<0.001$ \\
SOD & $228.7(72.52)$ & $214.3(90.97)$ & $221.5(124.23)$ & 0.749 \\
\hline
\end{tabular}

${ }^{a}$ Statistically significant difference between control group and paracetamol group $(\mathrm{p}<0.001)$; ${ }^{\mathrm{b}}$ Statistically significant difference between control group and clchicine group $(\mathrm{p}<0.001)$.

Table 5. Comparison of pre-treatment and post-treatment oxidant and antioxidant levels of the patient groups.

\begin{tabular}{|c|c|c|c|c|c|}
\hline Variables & Pre-treatment (mean(SD)) & Post-treatment (mean(SD)) & $\mathrm{p}$-Value ${ }^{\mathrm{a}}$ & Change (mean(SD)) & p-Value ${ }^{b}$ \\
\hline Catalase & & & & & 0.738 \\
\hline Paracetamol & $54.8(13.02)$ & $60.2(16.52)$ & 0.645 & $-0.09(20.42)$ & \\
\hline Colchicine/Par & $59.2(18.97)$ & $62.1(16.62)$ & 0.866 & $0.47(20.32)$ & \\
\hline GSH & & & & & 0.560 \\
\hline Paracetamol & $1.0(0.17)$ & $1.0(0.12)$ & 0.949 & $0.01(0.14)$ & \\
\hline Colchicine/Par & $0.99(0.06)$ & $1.0(0.07)$ & 0.271 & $0.01(0.11)$ & \\
\hline TAC & & & & & 0.206 \\
\hline Paracetamol & $24.0 \pm 7.25$ & $24.6 \pm 7.10$ & 0.754 & $0.6 \pm 10.32$ & \\
\hline Colchicine/Par & $21.6 \pm 5.31$ & $25.3 \pm 5.55$ & 0.018 & $3.7 \pm 7.67$ & \\
\hline MDA & & & & & 0.817 \\
\hline Paracetamol & $6.6(1.32)$ & $5.6(1.24)$ & 0.002 & $-0.64(1.87)$ & \\
\hline Colchicine/Par & $6.1(1.94)$ & $5.6(0.84)$ & 0.014 & $-0.83(2.22)$ & \\
\hline SOD & & & & & 0.727 \\
\hline Paracetamol & $214.3(90.97)$ & $228.7(72.52)$ & 0.610 & $20.4(98.87)$ & \\
\hline Colchicine/Par & $221.5(124.23)$ & $221.5(95.15)$ & 0.696 & $34.3(109.37)$ & \\
\hline
\end{tabular}

${ }^{a}$ Comparisons between pre-treatment and post-treatment within the same groups, according to the Bonferroni correction $\mathrm{p}$ value $<0.025$ was considered statistically significant for the results; ${ }^{\mathrm{b}}$ Comparisons between the groups in terms of treatment-related change, $\mathrm{p}$ value $<0.05$ was considered statistically significant for the results. 
$[3,4]$.

These crystals induce and maintain inflammation and ROS production in osteoarthritic joint and hence degredate more and more cartilage and produce pain and finally joint impairment occurs. To the best of our knowledge this is the first study in the literature evaluates colchicine effects on whole blood oxidant load and antioxidant levels in patients with knee OA. Our study showed that colchicine lowers whole blood MDA which is a lipid peroxidation compound and elevates TAC levels in patients with knee OA. Namely colchicine has antioxidant activity and also enhances total antioxiant capacity. However in this study, colchicine has no effect on whole blood SOD, CAT enzyme activities and GSH levels in patients with knee OA.

The current treatment of OA is primarily focused on relief of the symptoms by use of rapid action drugs (analgesics, cycloxygenase (COX-2) specific inhibitors and NSAIDS). These drugs do not effect the underlying pathogenesis of OA, thus have minimal role in modifying disease course and improving quality of life $[35,36]$.

In conclusion both paracetamol $3000 \mathrm{mg} /$ day and 3000 $\mathrm{mg}$ paracetamol plus $1.5 \mathrm{gr} /$ day colchicine is effective in the treatment of patients with knee osteoarthritis. But the addition of colchicine to paracetamol produced significantly greater symptomatic benefit than paracetamol alone. Our study also showed that colchicine lowers whole blood MDA which is a lipid peroxidation compound and elevates TAC levels in patients with knee OA. This may show probable disease modifying effect of colchicine in knee osteoarthritis which require further long period laboratory and radiologic investigations.

\section{Acknowledgements}

This research was funded by Yuzuncu Yil University Science Researchs Supporting Agency.

\section{REFERENCES}

[1] M. B. Goldring and S. R. Goldring, "Osteoarthritis," Journal of Cellular Physiology, Vol. 213, No. 3, 2007, pp. 626-634. doi:10.1002/jep.21258

[2] P. Sarzi-Puttini, M. A. Cimmino, R. Scarpa, R. Caporali, F. Parazzini, A. Zaninelli, F. Atzeni and B. Canesi, "Osteoarthritis: An Overview of the Disease and İts Treatment Strategies," Seminars in Arthritis and Rheumatism, Vol. 35, No. 1, 2005, pp. 1-10.

[3] Y. Z. Liu, A. P. Jackson and S. D. Cosgrove, "Contribution of Calcium-Containing Crystals to Cartilage Degradation and Synovial İnflammation in Osteoarthritis," Osteoarthritis and Cartilage, Vol. 17, No. 10, 2009, pp. 1333-1340.

[4] K. Jaovisidha and A. K. Rosenthal, "Calcium Crystals in Osteoarthritis," Current Opinion in Rheumatology, Vol. 14, No. 3, 2002, pp. 298-302.
[5] H. K. Ea and F. Liote, "Advances in Understanding Calcium-Containing Crystal Disease," Current Opinion in Rheumatology, Vol. 21, No. 2, 2009, pp. 150-157.

[6] E. A. Regan, R. P. Bowler and J. D. Crapo, "Joint Fluid Antioxidants Are Decreased in Osteoarthritic Joints Compared to Joints with Macroscropically İntact Cartilage and Subacute İnjury," Osteoarthritis Cartilage, Vol. 16, No. 4, 2008, pp. 515-521.

[7] O. Altindag, O. Erel, N. Aksoy, S. Selek, H. Celik and M. Karaoglanoglu, "Increased Oxidative Stress and Its Relation with Collagen Metabolism in Knee Osteoarthritis," Rheumatology International, Vol. 27, No. 4, 2007, pp. 339-344.

[8] S. Sarban, A. Kocyigit, M. Yazar and U. E. Isıan, "Plasma Total Antioxidant Capacity, Lipid Peroxidation, and Erythrocyte Antioxidant Enzyme Activities in Patients with Rheumatoid Arthritis and Osteoarthritis," Clinical Biochemistry, Vol. 38, No. 11, 2005, pp. 981-986. doi:10.1016/j.clinbiochem.2005.08.003

[9] A. F. Chen, C. M. Davies, M. De Lin and B. Fermor, "Oxidative DNA Damage in Osteoarthritic Porcine Articular Cartilage," Journal of Cellular Physiology, Vol. 217, No. 3, 2008, pp. 828-833. doi:10.1002/jcp.21562

[10] C. M. Davies, F. Guilak, J. B. Weinberg and B. Fermor, "Reactive Nitrogen and Oxygen Species in İterleukin1-Mediated DNA Damage Associated with Osteoarthritis," Osteoarthritis Cartilage, Vol. 16, No. 5, 2008, pp. 624630. doi:10.1016/j.joca.2007.09.012

[11] Y. E. Henrotin and B. Kurz, "Oxygen and Reactive Oxygen Species in Cartilage Degradation: Friends or Foes?" Osteoarthritis Cartilage, Vol. 13, No. 8, 2005, pp. 643654.

[12] J. C. Monboisse and J. P. Borel, "Oxidative Damage to Collagen," EXS, Vol. 62, 1992, pp. 323-327.

[13] J. M. McCord, "Free Radicals and İnflammation: Protection of Synovial Fluid by Superoxide Dismutase," Science, Vol. 185, No. 150, 1974, pp. 529-531.

[14] A. Klamfeldt and S. Marklund, "Enhanced Breakdown in Vitro of Bovine Articular Cartilage Proteoglycans by Conditional Synovial Medium: The Effect of Superoxide Dismutase and Catalase," Scandinavian Journal of Rheumatology, Vol. 16, 1987, pp. 41-45.

[15] H. Burkhardt, M. Schwingel, H. Menninger, H. W. Macartney and H. Tschesche, "Oxygen Radicals as Effectors of Cartilage Destruction: Direct Degradative Effect on Matrix Components and İndirect Action via Activation of Latent Collagenase from Polymorphonuclear Leukocytes," Arthritis \& Rheumatism, Vol. 29, 1986, pp. 379-387. doi:10.1002/art.1780290311

[16] H. M. Lander, A. J. Milbank, J. M. Tauras, D. P. Hajjar, B. L. Hempstead, G. D. Schwartz, et al., "Redox Regulation of Cell Signalling," Nature, Vol. 381, 1996, pp. 380-381. doi:10.1038/381380a0

[17] R. H. Burdon, "Superoxide and Hydrogen Peroxide in Relation to Mammalian Cell Proliferation," Free Radical Biology \& Medicine, Vol. 18, 1995, pp. 775-794. doi:10.1016/0891-5849(94)00198-S 
[18] F. J. Blanco, R. L. Ochs, H. Schwarz and M. Lotz, "Chondrocyte Apoptosis İnduced by Nitric Oxide," American Journal of Pathology, Vol. 146, No. 1, 1995, pp. 75-85.

[19] S. B. Abramson, M. Attur, A. R. Amin and R. Clancy, "Nitric Oxide and İnflammatory Mediators in the Perpetuation of Osteoarthritis," Current Rheumatology Reports, Vol. 3, No. 6, 2001, pp. 535-541.

[20] D. Taskiran, M. Stefanovic-Racic, H. Georgescu and C. Evans, "Nitric Oxide Mediates Suppression of Cartilage Proteoglycan Synthesis by İnterleukin-1," Biochemical and Biophysical Research Communications, Vol. 200, No. 1, 1994, pp. 142-148.

[21] Y. Hirai, K. Migita, S. Honda, Y. Ueki, S. Yamasaki and S. Urayama, et al., "Effects of Nitric Oxide on Matrix Metalloproteinase-2 Production by Rheumatoid Synovial Cells," Life Sciences, Vol. 68, No. 8, 2001, pp. 913-920. doi:10.1016/S0024-3205(00)00998-X

[22] R. M. Clancy, S. B. Abramson, C. Kohne and J. Rediske, "Nitric Oxide Attenuates Cellular Hexose Monophosphate Shunt Response to Oxidants in Articular Chondrocytes and Acts to Promote Oxidant İnjury," Journal of Cellular Physiology, Vol. 172, No. 2, 1997, pp. 183-191.

[23] N. Alaaeddine, J. A. Di Battista, J. P. Pelletier, J. M. Cloutier, K. Kiansa, et al., "Osteoarthritic Synovial Fibroblasts Possess an İncreased Level of Tumor Necrosis Factor-Receptor 55 (TNF-R55) That Mediates Biological Activation by TNF-Alpha," Journal of Rheumatology, Vol. 24, No. 10, 1997, pp. 1985-1994.

[24] M. Lotz, "The Role of Nitric Oxide in Articular Cartilage Damage," Rheumatic Disease Clinics of North America, Vol. 25, No. 2, 1999, pp. 269-282.

[25] A. Anandacoomarasamy and L. March, "Current Evidence for Osteoarthritis Treatments," Therapeutic Advances in Musculoskeletal Disease, Vol. 2, No. 1, 2010, pp. 17-28.

[26] M. Fajardo and P. E. Di Cesare, "Disease-Modifying Therapies for Osteoarthritis: Current Status," Drugs Aging, Vol. 22, No. 2, 2005, pp. 141-161. doi:10.2165/00002512-200522020-00005

[27] M. E. Zagaria, "Osteoarthritis in Seniors," US Pharmacist, Vol. 31, No. 1, 2006, pp. 20-24.

[28] S. K. Das, S. Ramakrishnan, K. Mishra, et al., "A
Randomized Controlled Trial to Evaluate the Slow-Acting Symptom-Modifying Effects of Colchicine in Osteoarthritis of the Knee: A Preliminary Report," Arthritis \& Rheumatism, Vol. 47, No. 3, 2002, pp. 280-284. doi:10.1002/art.10455

[29] M. Modriansky, Y. Y. Tyurina, V. A. Tyurin, T. Matsura, A. A. Shvedova, J. C. Yalowich and V. E. Kagan, "Anti-/ Pro-Oxidant Effects of Phenolic Compounds in Cells: Are Colchicine Metabolites Chain-Breaking Antioxidants?" Toxicology, Vol. 177, No. 1, 2002, pp. 105-117. doi:10.1016/S0300-483X(02)00199-3

[30] D. Das, P. W. Pemberton, P. C. Burrows, C. Gordon, A. Smith, R. F. McMahon and T. W. Warnes, "Antioxidant Properties of Colchicine in Acute Carbon Tetrachloride İnduced Rat Liver İnjury and İts Role in the Resolution of Established Cirrhosis," Biochimica et Biophysica Acta, Vol. 1502, 2000, pp. 351-362. doi:10.1016/S0925-4439(00)00059-4

[31] E. W. Chia, R. Grainger and J. L. Harper, "Colchicine Suppresses Neutrophil Superoxide Production in a Murine Model of Gouty Arthritis: A Rationale for Use of Low-Dose Colchicine," British Journal of Pharmacology, Vol. 153, No. 6, 2008, pp. 1288-1295.

[32] E. H. Tüzün, L. Eker, A. Aytar, A. Daşkapan and M. Bayramoğlu, "Acceptability, Reliability, Validity and Responsiveness of the Turkish Version of WOMAC Osteoarthritis Index," Osteoarthritis Cartilage, Vol. 13, No. 1, 2005, pp. 28-33. doi:10.1016/j.joca.2004.10.010

[33] K. R. Hegde, M. G. Henein and S. D. Varma, "Establishment of Mouse as an Animal Model for Study of Diabetic Cataracts: Biochemical Studies," Diabetes, $\mathrm{Ob}$ esity and Metabolism, Vol. 5, No. 2, 2003, pp. 113-119.

[34] L. Ediz and I. Tekeoğlu, "Symptom Modifying Effect of Colchicine in Patients with Knee Osteoarthritis," Journal of Clinical and Analytical Medicine, Vol. 3, No. 1, 2012, pp. 63-67. doi:10.4328/JCAM.563

[35] A. Mahajan, S. Verma and V. Tandon, "Osteoarthritis," Journal of the Association of Physicians of India, Vol. 53, 2005, pp. 634-641.

[36] P. R. Colville-Nash and D. A. Willoughby, "COX-1, COX-2 and Articular Joint Disease: A Role of Chondroprotective Agents," Biorheology, Vol. 39, No. 1-2, 2002, pp. 171-179. 